\title{
Management of a Traumatized Open Apex Tooth with a Combination of Mineral Trioxide Aggregate Apical Plug and Platelet-rich Fibrin Apical Matrix
}

\author{
${ }^{1}$ Ritesh Balasaheb Pawar, ${ }^{2}$ Sumanthini V Margsahayam, ${ }^{3}$ Vanitha U Shenoy, ${ }^{4}$ Saimanaaz Abdul Hakeem Shaikh
}

\begin{abstract}
The absence of a natural apical constriction in permanent tooth makes endodontic treatment a challenge. Traditionally, calcium hydroxide was used for inducing the formation of an apical barrier. Currently, mineral trioxide aggregate (MTA) has shown promising results for apexification procedures. In an open apex, it is imperative to limit the MTA placement within the confines of the root canal for predictable healing. The placement of an internal matrix may limit the extrusion to some extent. Many materials can be used as internal matrix such as collagen membrane, calcium sulphate, hydroxyapatite, freeze dried bone, and platelet-rich fibrin (PRF) among others. This case report presents a successful demonstration of the management of an open apex using MTA placed over an internal apical matrix of PRF.
\end{abstract}

Keywords: Apexification, Mineral trioxide aggregate, Open apex, Platelet-rich fibrin.

How to cite this article: PawarRB, Margsahayam SV, ShenoyVU, Shaikh SAH. Management of a Traumatized Open Apex Tooth with a Combination of Mineral Trioxide Aggregate Apical Plug and Platelet-rich Fibrin Apical Matrix. J Contemp Dent 2016;6(1):57-62.

Source of support: Nil

Conflict of interest: None

\section{INTRODUCTION}

Traumatic injuries to teeth in young children may lead to pulp necrosis and root development may cease subsequently resulting in the formation of an open apex. An open apex may also result from extensive resorption of a mature apex following orthodontic treatment, or due to periradicular inflammation. ${ }^{1}$ Teeth with open apex are a challenge to manage owing to the difficulties in debriding, disinfecting, and predictable sealing of the root canal space. As the apex is not completely formed, no barrier,

\footnotetext{
${ }^{1,4}$ Postgraduate Student, ${ }^{2}$ Professor, ${ }^{3}$ Professor and Head

${ }^{1-4}$ Department of Conservative Dentistry and Endodontics MGM Dental College and Hospital, Navi Mumbai, Maharashtra India
}

Corresponding Author: Ritesh Balasaheb Pawar, Postgraduate Student, Department of Conservative Dentistry and Endodontics MGM Dental College, Navi Mumbai, Maharashtra, India, Phone: 8879923257, e-mail: riteshppawar24688@gmail.com exists to stop the obturating material from moving into and traumatizing the apical periodontal tissues. Thin dentinal walls are susceptible to fracture both during and after treatment. These problems are overcome by stimulating the formation of a hard tissue barrier to allow for optimal filling of the canal and reinforcing the weakened root against fracture both during and after apexification. The process of creating a hard tissue barrier at an open apex or at a grossly over-instrumented apex is termed as apexification. Calcium hydroxide $(\mathrm{CH})$ has been the material of choice for inducing formation of hard tissue barrier. ${ }^{2}$ Calcific barrier formation is induced with repeated changes of material over the course of 5 to 20 months $^{3}$ averaging 12.9 months. ${ }^{4}$ Disadvantages of this technique are its prolonged treatment time, the need for multiple visits, reduced fracture resistance with prolonged use of $\mathrm{CH}$, radiographs, the loss of coronal restoration making the tooth susceptible to reinfection, and patient complaints. Mineral trioxide aggregate (MTA) has been used as an alternative to $\mathrm{CH}$ apexification to seal the open apex..$^{5}$ The favorable properties of MTA include biocompatibility, good sealing ability, and promote periradicular tissue regeneration. ${ }^{6}$

In wide open apex, it is difficult to place MTA and confine it within the canal. The placement of an apical matrix will limit the MTA within the confines of the root canal space; furthermore, it will aide in obtaining a well-compacted apical plug. Lemon ${ }^{7}$ developed the internal matrix concept, in which an intermediate layer of hydroxyapatite was placed through the perforation to form an external barrier and matrix, against which the perforation repair material (amalgam) could be condensed. Bargholz ${ }^{8}$ introduced "modified matrix concept" in which a resorbable collagen was placed as a matrix followed by condensation of MTA, thereby preventing MTA overfill. Several other materials have also been recommended to create a matrix in cases of perforations as well as teeth with incomplete formation of apex, which include $\mathrm{CH}$, hydroxyapatite, resorbable collagen, calcium sulphate, platelet-rich fibrin (PRF), freeze dried bone allograft and tricalcium phosphate. ${ }^{9-12}$

Platelet-rich fibrin developed in France by Choukroun and Dohan represents a new step in the platelet gel therapeutic concept. Platelet-rich fibrin is a matrix of 
autologous fibrin embedded with a large quantity of platelet and leukocyte cytokines during centrifugation. It is interesting to know that PRF matrix enmeshes glycosaminoglycans from blood and platelet. Glycosaminoglycans have a strong affinity with small circulating peptides and a great capacity to support cell migration and healing processes..$^{12}$ The PRF membrane has shown promising apical matrix for MTA. It prevents extrusion of MTA and promotes wound healing.

This case report describes the nonsurgical management of a traumatized maxillary central incisor with a wide open apex by placing MTA in combination with autologous PRF membrane as an apical matrix.

\section{CASE REPORT}

A 32-year-old male patient was referred to the Department of Conservative Dentistry and Endodontics with a chief complaint of fractured and discolored maxillary left central incisor (Fig. 1). Patient gave a history of trauma to maxillary left central incisor at the age of 10 years, following which the patient claimed that he had undergone dental treatment for the same. Medical history was noncontributory. Intraoral examination revealed a discolored maxillary left central incisor with temporary restoration in the access cavity. Patient had pain on percussion, periodontal probing within normal limits $(<3 \mathrm{~mm})$, and no mobility. Radiographic examination revealed an immature apex associated with a periapical lesion in relation to 21 (Fig. 1). A diagnosis of previously initiated root canal treatment with symptomatic apical periodontitis in relation to 21 was arrived at.

The treatment options given to the patient were nonsurgical endodontic treatment with one-step MTA apexification procedure, endodontic treatment followed by periapical surgery for removal of the lesion and retrofilling, extraction, and single tooth implant or fixed partial denture. Considering the age of the patient and the surgical trauma that could occur, it was decided to opt for nonsurgical root canal treatment. The tooth was isolated under rubber dam, temporary restoration was removed, and endodontic access cavity was modified. The root canal was explored with a \#25 K-file (Mani, Tamil Nadu, India). The canal was patent, wide, and the largest file to bind to apical extent of the canal was \#120 K-file. An intraoral periapical radiograph (IOPA) was taken to determine the working length (Fig. 1B). The root canal was gently, circumferentially filed with \#120 K-file. The canal was irrigated intermittently with $2.5 \%$ sodium hypochlorite $(\mathrm{NaOCl})$ (Prime Dental Products Pvt Ltd., Maharashtra, India). Finally, passive ultrasonic irrigation was carried with ultrasonic \#25K-file (Satellac Acteon Group, New Delhi, India) at a setting of 4.0 for
1 minute with $\mathrm{NaOCl}$ as irrigant. The root canal was then dried with sterile absorbent points (Sure endo; SureDent Co. Ltd., Gyeonggi-do, Korea). A thick paste of CH (Deepashree Products, Ratnagiri, India) mixed with saline was placed in the root canal (Fig. 2), and the patient was recalled after 2 weeks. At 2-week recall appointment, $\mathrm{CH}$ was removed using H-files (Mani, Tamil Nadu, India) and passive ultrasonic irrigation. The root canal was then dried with sterile absorbent points.

It was decided to use PRF membrane as an internal matrix at the apical foramen prior to MTA placement. Ten milliliters of patient's venous blood was drawn by venipuncture from the anticubital vein, and was collected in $10 \mathrm{ml}$ test tube without anticoagulant and immediately centrifuged (Process ${ }^{\circledR}$ centrifuge PC-02; Process Ltd., Nice, France) at 3000 rpm for 15 minutes. ${ }^{13}$ The centrifugation product consisted of three layers
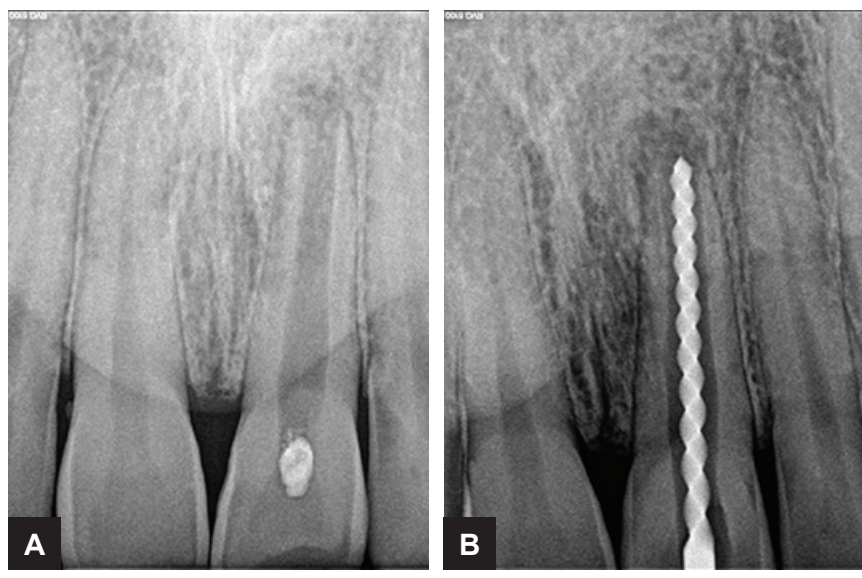

Figs 1A and B: (A) Preoperative radiograph showing 21 with access cavity restoration associated with an open apex and periapical radiolucency and $(B)$ working length radiograph

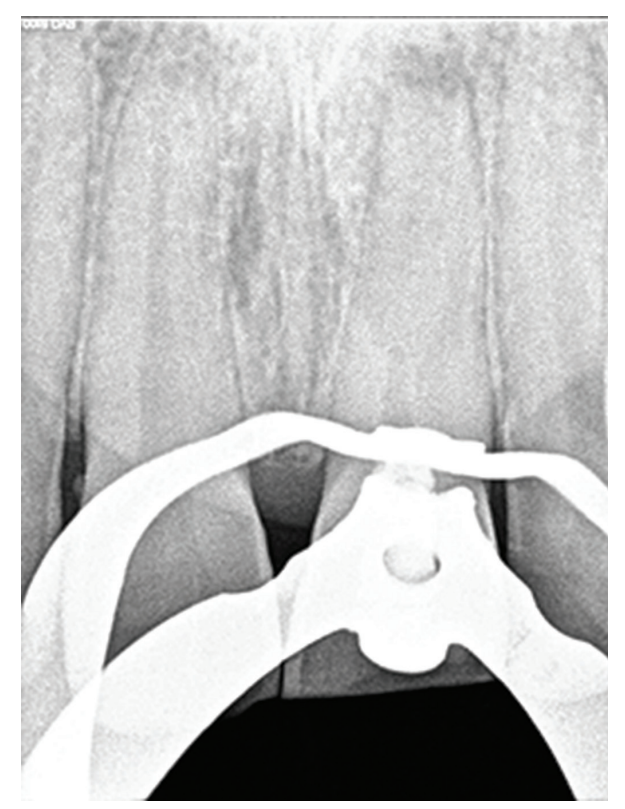

Fig. 2: Intraoral periapical radiograph showing dense calcium hydroxide medication placed in 21 


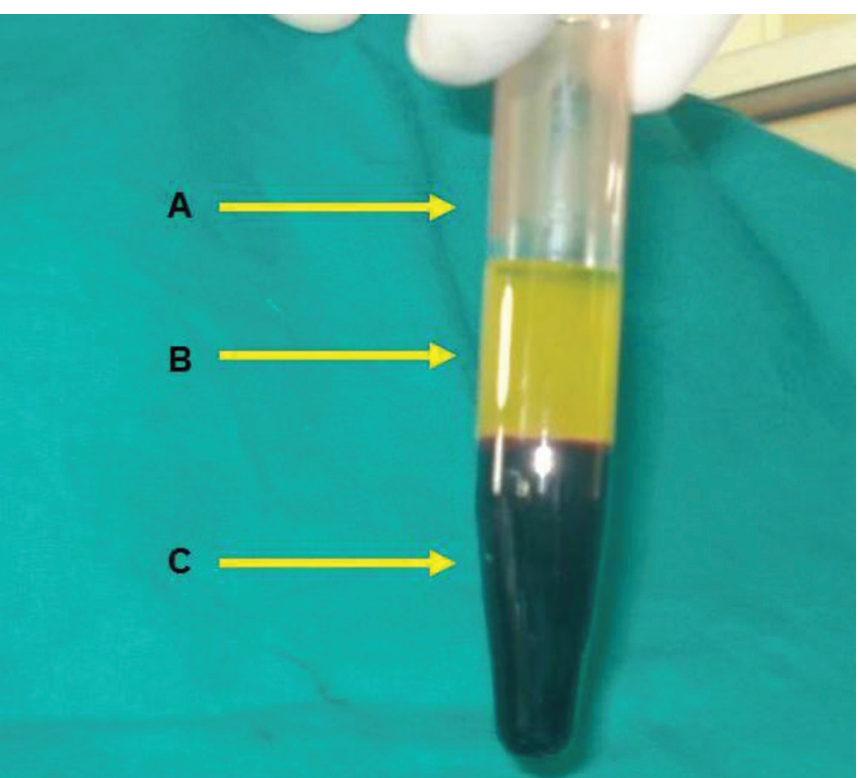

Fig. 3: Centrifuged blood showing three layers: A: Top—plateletpoor plasma; B: Middle_-PRF; C: bottom layer containing RBCs

(Fig. 3): A base of red blood corpuscles (RBCs) at the bottom, acellular plasma on the surface, and PRF clot in the middle. The fibrin clot was separated from the lower most layer of the centrifugation product. The PRF clot was gently pressed into a membrane form with a sterile moist gauze (Fig. 4) and placed on a sterile glass slab. The required quantity of PRF membrane was introduced into the canal and positioned apically with a hand plugger (GDC, Punjab, India) at the apical foramen and into the bony space beyond it. White Proroot MTA (Dentsply, Ballaigues, Switzerland) was mixed with sterile water as per manufacturer instructions and placed in the apical third using hand pluggers and compacted with absorbent points. It was further com-

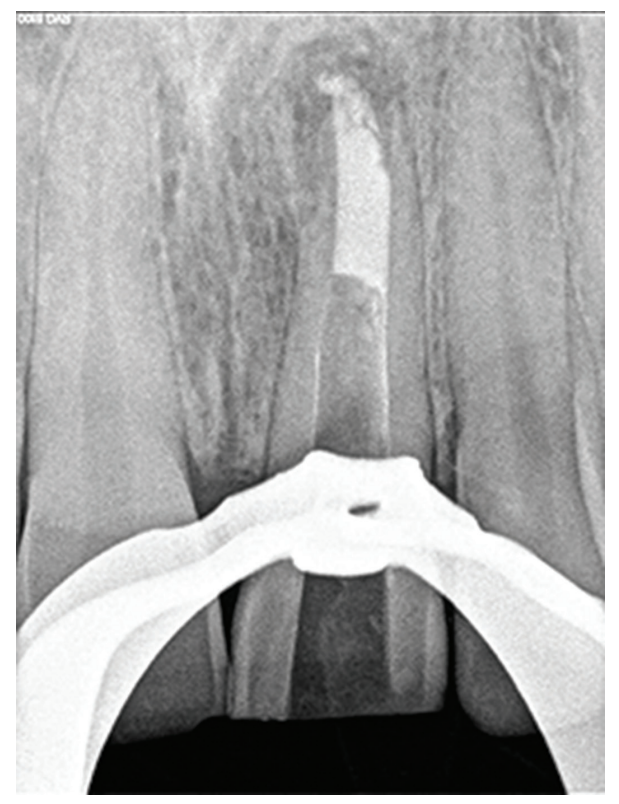

Fig. 5: Mineral trioxide aggregate apical plug

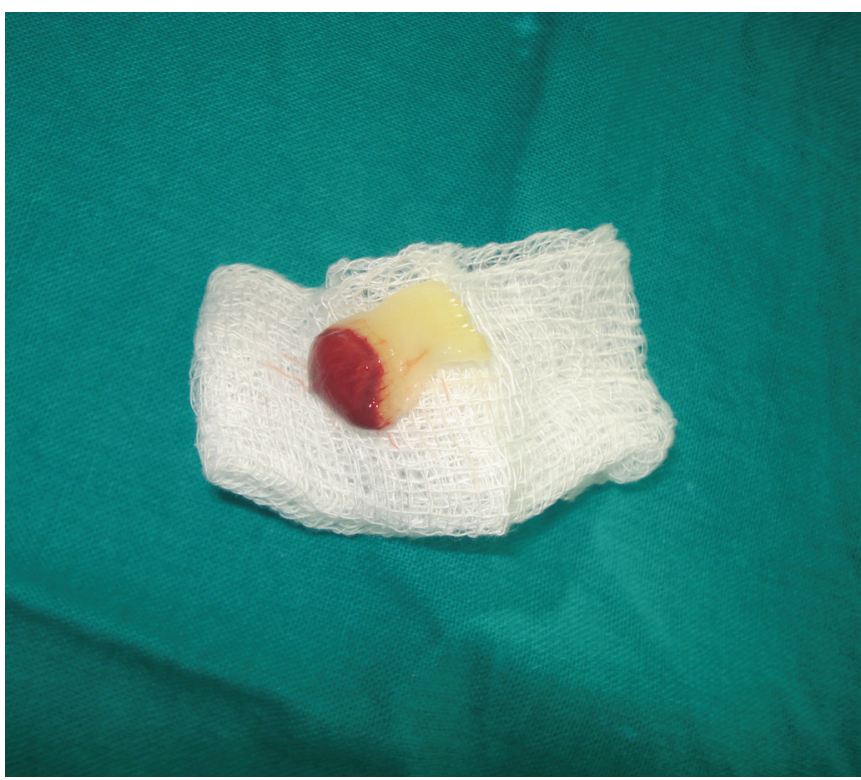

Fig. 4: Platelet-rich fibrin membrane in gauze

pacted using indirect ultrasonics. Thus, $5 \mathrm{~mm}$ of MTA apical plug was obtained, and a radiograph was taken to confirm the placement of dense MTA apical plug (Fig. 5). A moistened pellet of cotton was placed in the canal and access cavity was sealed with Cavit (3M ESPE, Seefeld, Germany).

Patient was recalled after 2 days and was completely asymptomatic. Temporary restoration was removed and the set of MTA was confirmed. The canal was irrigated with $2.5 \% \mathrm{NaOCl}$ followed by saline, dried, and a prefabricated fiber post (Luminex; Dentatus AB, Stockholm, Sweden) was cemented using Paracore (Coltene/whaldent Inc., Cuyahoga Falls, $\mathrm{OH}$, USA) (Fig. 6). Further, the tooth was restored

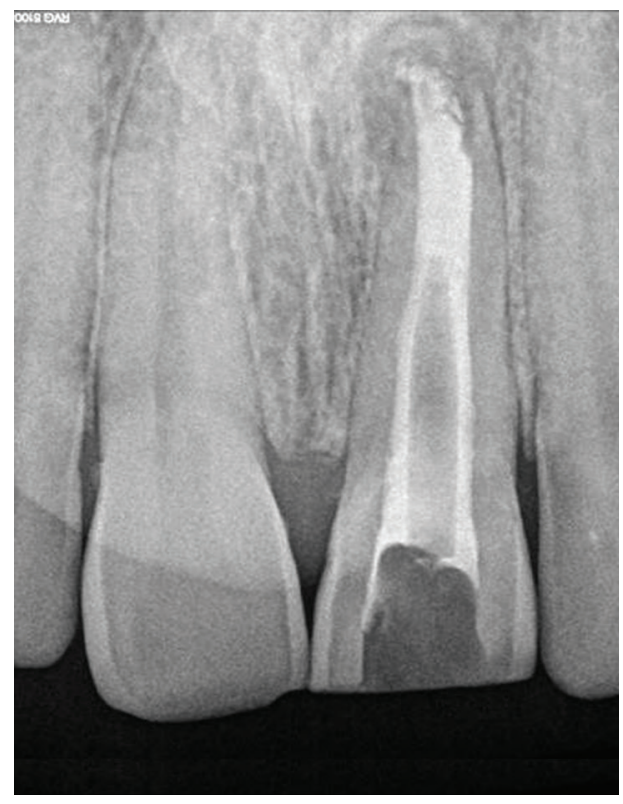

Fig. 6: Fiber post luted with paracore 


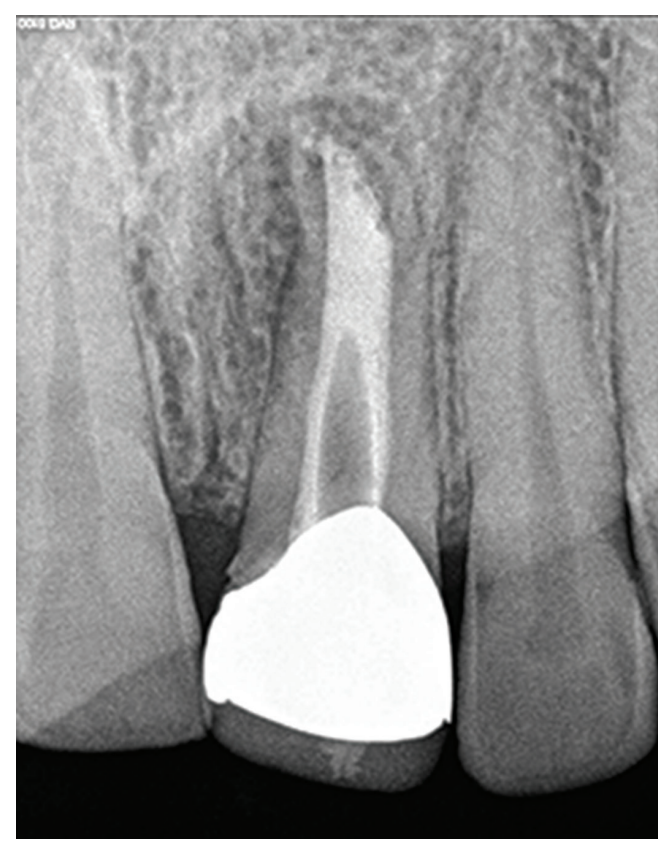

Fig. 7: Three-month radiograph

with porcelain fused to metal (PFM) crown. The patient was recalled after 3 months (Fig. 7). At 9 months of follow-up, the patient was asymptomatic and periapical radiograph demonstrated satisfactory healing (Fig. 8).

\section{DISCUSSION}

Root-end closure previously referred to as apexification is the induction of an artificial calcified barrier across the open apex. An immature root has a considerably wide apical opening that may be apically diverging or parallel with thin canal walls. The factors essential for success are thorough debridement and disinfection of the root canal space. The presence of fragile radicular walls precludes optimal filing as carried out in teeth with mature apices. Hence, disinfection of the root canal system relies mainly on the action of irrigants and intracanal medications. Passive ultrasonic irrigation was done in the canal space, as it is more effective than conventional syringe irrigation at eliminating debris, bacteria, and pulp tissue in the isthmuses, lateral and apical ramifications, and flattened areas. ${ }^{1,14}$ In the present case, $\mathrm{CH}$ paste was placed to disinfect the canal that would create an environment conducive for the formation of an apical barrier. ${ }^{15}$

Different materials have been used for root-end closure and the most promising being MTA. Due to its noncytotoxicity, MTA has good biological properties and stimulates repair. ${ }^{15}$ The major problem in cases of a wide open apex is confining MTA to the canal space while obtaining a void-free apical plug during compaction, thereby avoiding the extrusion of a large amount of MTA

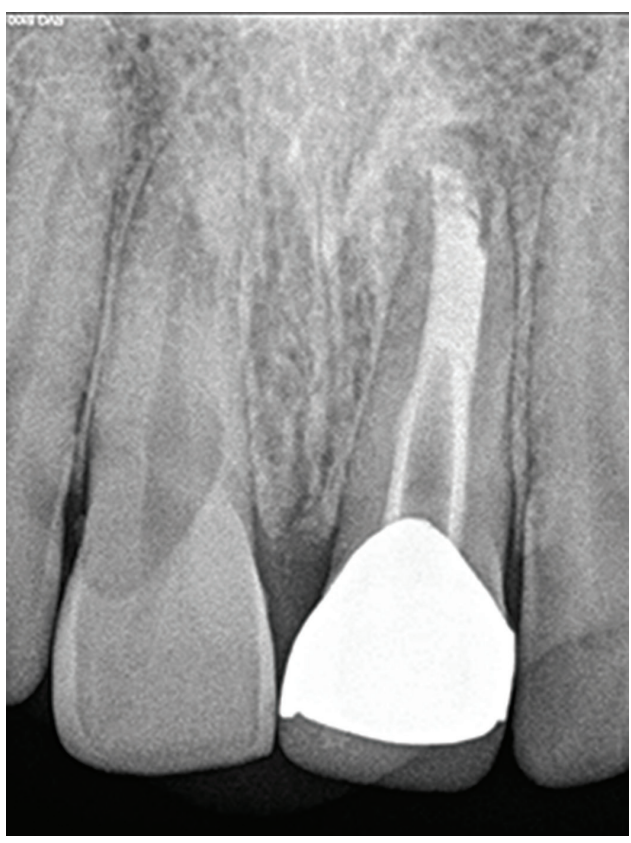

Fig. 8: Nine-month radiograph showing satisfactory healing

into the periapical tissue. All types of MTA material contain arsenic that might leach out into the periapical tissues. Although the quantity released is insignificant it can potentially cause toxicity, which has been a cause of concern. ${ }^{16}$ Moreover, a large volume of extruded material may set before it disintegrates and gets resorbed. This might result in persistent inflammatory process, which may complicate or even prevent the repair of the tissue ${ }^{17}$ Hence, the internal matrix is placed to prevent extrusion of the material. Extent of periapical bone loss significantly influences the clinical decision for the need and also type of internal matrix. Moreover, the type of internal matrix to be placed also depends on the apical foramen diameter and facilitates the placement of a dense apical plug, thereby allowing a favorable healing of the periodontal tissues.

Various materials have been reported to be used as an internal apical barrier in combination with MTA. ${ }^{18}$ The currently introduced technique of using PRF membrane was considered as an apical matrix. Platelet-rich fibrin is an autologous fibrin gel with cicatricial properties; it is a new platelet concentrate, which has advantages of low cost, ease of procedure, does not dissolve quickly after application, and is biocompatible. The success of this technique depends entirely on the speed of blood collection and transfer to the centrifuge, if prolonged failure will occur. A large quantity of platelets and leukocyte cytokines are embedded during centrifugation. ${ }^{12}$ The intrinsic incorporation of cytokines within the fibrin mesh allows for their progressive release over time (7-10 days), as the network of fibrin disintegrates. The use of this platelet and immune concentrate offers 
the following advantages. ${ }^{19}$ First, the fibrin clot plays an important mechanical role, with the PRF membrane maintaining and protecting the grafted biomaterials and PRF fragments serving as biological connectors between bone particles. Second, the integration of this fibrin network into the regenerative site facilitates cellular migration, particularly for endothelial cells necessary for the neo-angiogenesis, ${ }^{20}$ vascularization, and survival of the graft. Third, the platelet cytokines (platelet-derived growth factor, transforming growth factor, insulin-like growth factor-1) are gradually released as the fibrin matrix is resorbed, thus creating a perpetual process of healing. Lastly, the presence of leukocytes and cytokines in the fibrin network can play a significant role in the selfregulation of inflammatory and infectious phenomena within the grafted material.

Although one-step MTA apexification provides several advantages, the canal wall remains thin, weak, and susceptible to fracture. A fiber post was cemented with dual cure composite resin. Studies have suggested that the fiber posts significantly reinforce the cervical area of the teeth and decrease catastrophic fracture. This might be related to a similar modulus of elasticity of fiber post and dentin. Also, fiber post might evenly distribute forces along the root. ${ }^{21}$

\section{CONCLUSION}

This case report demonstrates that teeth with wide open apices can be treated successfully with nonsurgical treatment using PRF as internal apical matrix under MTA apical plug for one-step apexification procedure. Further long-term randomized clinical trial need to be conducted to evaluate the successful outcomes.

\section{CLINICAL SIGNIFICANCE}

In teeth with immature apex, a biodegradable, autologous material, such as PRF membrane can be placed as barrier prior to compacting MTA in the apical third of the root canal. This will aid in the prevention of extrusion of MTA and enhance the healing potential.

\section{REFERENCES}

1. Walton R, Torabinejad M. Principles and practice of endodontics. 3rd ed. Philadelphia: WB Saunders Company; 2002. p. 388.

2. Rafter M. Apexification: a review. Dent Traumatol 2005 Feb; 21(1):1-8.

3. Sheehy EC, Roberts GJ. Use of calcium hydroxide for apical barrier formation and healing in nonvital immature permanent teeth: a review. Br Dent J 1997 Oct 11;183(7): 241-246.
4. Dominguez RA, Munoz L, Aznar T. Study of calcium hydroxide apexification in 26 young permanent incisors. Dent Traumatol 2005 Jun;21(3):141-145.

5. Sumanthini MV, Naisargi S, Malgaonkar M. Management of non-vital maxillary central incisors with open apex using mineral trioxide aggregate apical plugs-case report. J Contemp Dent 2011;1:40-43.

6. Simon S, Rilliard F, Berdal A, Machtou P. The use of mineral trioxide aggregate in onevisit apexification treatment: a prospective study. Int Endod J 2007 Mar;40(3):186-197.

7. Lemon RR. Nonsurgical repair of perforation defects. Internal matrix concept. Dent Clin North Am 1992 Apr;36(2):439-457.

8. Bargholz C. Perforation repair with mineral trioxide aggregate: a modified matrix concept. Int Endod J 2005 Jan;38(1): 59-69.

9. Rafter M, Baker M, Alves M, Daniel J, Remeikis N. Evaluation of healing with use of an internal matrix to repair furcation perforations. Int Endod J 2002 Sep;35(9):775-783.

10. Alhadainy HA, Himel VT, Lee WB, El Baghdady YM. Use of a hydroxylapatite-based material and calcium sulfate as artificial floor to repair furcal perforations. Oral Surg Oral Med Oral Pathol Oral Radiol Endod 1998 Dec;86(6): 723-729.

11. Alves FR, Almeida BM, Neves MA, Moreno JO, Rocas IN, Siqueira JF Jr. Disinfecting oval-shaped root canals: Effectiveness of different supplementary approaches. J Endod 2011 Apr;37(4):496-501.

12. Bodhawani M, Shenoy V, Sumanthini M. Mineral trioxide aggregate apexification in non vital immature central incisor tooth using internal matrix. J Contemp Dent 2014;4: 113-117.

13. Dohan DM, ChokrounJ, Diss A, Dohan SL, Dohan AJ, MouhyiJ, Gogly B. Platelet-rich fibrin a second generation platelet concentrate- part I: technological concept and evolution. Oral Surg Oral Med Oral PatholOral Radiol Endod 2006 Mar;101(3):e37-e44.

14. Al-Jadaa A, Paque F, Attin T, Zehnder M. Necrotic pulp tissue dissolution by passive ultrasonic irrigation in simulated accessory canals: impact of canal location and angulation. Int Endod J 2009 Jan;42(1):59-65.

15. Barbosa CAM, Goncalves RB, Siqueira JF, Uzeda M. Evaluation of the antibacterial activities of calcium hydroxide, chlorhexidine and camphorated paramonochlorophenol as intracanal medicament. A clinical and laboratory study. J Endod 1997 May;23(5):297-300.

16. Danesh G, Dammaschke T, Gerth HUV, Zandbiglari T, Schäfer E. A comparative study of selected properties of ProRoot mineral trioxide aggregate and two Portland cements. Int Endod J 2006 Mar;39(3):213-219.

17. Matt GD, Thorpe JR, Strother JM, McClanahan SB. Comparative study of white and gray mineral trioxide aggregate simulating a one- or two-step apical barrier technique. J Endod 2004 Dec;30(12):876-879.

18. Andreasen JO, Hjorting-Hansen E. Intra-alveolar root fractures: radiographic and histologic study of 50 cases. J Oral Surg 1967 Sep;25(5):414-426.

19. SimonpieriA,DelCorsoM,SammartinoG,DohanEhrenfestDM. The relevance of Choukroun's platelet-rich fibrin and metronidazole during complex maxillary rehabilitations using bone allograft. Part I: a new grafting protocol. Implant Dent 2009 Apr;18(2):102-111. 
20. SimonpieriA,DelCorsoM,SammartinoG,DohanEhrenfestDM. The relevance of Choukroun's platelet-rich fibrin and metronidazole during complex maxillary rehabilitations using bone allograft. Part II: implant surgery, prosthodontics, and survival. Implant Dent 2009 Jun;18(3):220-229.
21. Schmoldt SJ, Kirkpatrick TC, Rutledge RE, Yaccino JM. Reinforcement of simulated immature roots restored with composite resin, mineral trioxide aggregate, gutta-percha, or a fiber post after thermocycling. J Endod 2011 Oct;37(10): 1390-1393. 